\title{
ARTÍCULOS
}

Recibido 28.01.2013. Aprobado 29.11.2013

Evaluado por el sistema double blind review. Editor científico: Rodrigo Bandeira-de-Mello

DOI: http://dx.doi.org/10.1590/So034-759020140604

\section{CAPITAL INTELECTUAL Y COMPETENCIAS DISTINTIVAS EN PYMES MADERERAS DE ARGENTINA}

\author{
Capital intelectual e competências distintas em PMEs madeireiras da Argentina \\ Intellectual capital and distinctive skills in SMEs of the timber industry in Argentina
}

\begin{abstract}
RESUMEN
En la economía del conocimiento cada vez adquiere más importancia el capital intelectual como fuente de competencias distintivas. Una competencia distintiva se obtiene combinando los factores internos y externos de la empresa, mediante la estrategia, para generar mejor desempeño que sus competidores. Las pequeñas y medianas empresas (PyMEs) madereras argentinas suelen tener características específicas, de modo especial, aquellas relacionadas a su capital intelectual. En consecuencia se espera que el proceso de constitución de competencias distintivas sea diferente. Este trabajo analiza las particularidades de ese proceso. Los resultados obtenidos muestran como organizan sus recursos y capacidades para constituir su nivel de competitividad. Este genera mejores resultados organizativos, pero no afecta de modo directo a los resultados económicos.
\end{abstract}

PALABRAS CLAVE | Competencias distintivas, capital intelectual, resultados empresariales, PyMEs madereras, Argentina.

\section{RESUMO}

Na economia do conhecimento, o capital intelectual como fonte de competências distintas adquire cada vez mais importância. Uma competência distinta é obtida combinando os fatores internos e externos da empresa, mediante a estratégia, para gerar melhor desempenho do que seus concorrentes. As pequenas e médias empresas (PMEs) madeireiras argentinas podem ter características específicas, de modo especial, aquelas relacionadas com o seu capital intelectual. Em consequência, espera-se que o processo de constituição de competências distintas seja diferente. Este trabalho analisa as particularidades desse processo. Os resultados obtidos mostram como organizar seus recursos $e$ capacidades para constituir o seu nível de competitividade. Isto gera melhores resultados organizacionais, mas não afeta de modo direto os resultados econômicos.

PALAVRAS-CHAVE / Competências distintas, capital intelectual, resultados empresariais, PMEs madeireiras, Argentina.

CARLOS M. F-JARDON

cjardon@uvigo.es

Profesor del Departamento de

Economía Aplicada, Universidad de Vigo - Vigo, España

\section{MARÍA SUSANA MARTOS}

sumartos@gmail.com

Investigadora del Departamento

de Organización de Empresas,

Universidad de Vigo - Vigo, España

\begin{abstract}
In the knowledge economy, intellectual capital is increasingly becoming important as a source of core competences. A core competence is obtained by combining internal and external factors of the company by means of the strategy to generate better performance than its competitors. The Argentinian wood SMEs often have specific characteristics and, in particular, a limited intellectual capital. Consequently, it is expected that the process of establishing core competences is different. This paper analyzes the characteristics of that process. The results show how they organize their resources and capabilities to provide their competitiveness. This competitiveness leads to better organizational performance, but does not directly affect economic performance.
\end{abstract}

KEYWORDS / Core competences, intellectual capital, firm performance; wood industry SMEs, Argentina 


\section{INTRODUCCIÓN}

Las pequeñas y medianas empresas (PyMEs) son fundamentales en las economías modernas, puesto que generan una gran parte del empleo y del PIB. En particular, en Argentina, más del $99 \%$ de las empresas son pymes y generan más del $66 \%$ del empleo y más del $50 \%$ del PIB (Cohen Arazi, 2013). En este trabajo nos centramos en pymes madereras, es decir, pymes cuyo principal input es un recurso natural de la zona (la madera en este caso), o que se encuentran en la cadena de valor de ese input. Podemos incluirlas dentro del grupo de pymes basadas en recursos naturales (Giuliani, Pietrobelli, \& Rabellotti, 2005). Estas pymes presentan características específicas, tales como menos profesionalización (Angelelli, Moudry, \& Llisterri, 2006; Jardon, Gutawski, Martos, Aguilar, \& Barajas, 2007); más dificultades para obtener financiación (Idb \& Eri, 2008) y mayores dificultades de conseguir personal con formación (Zevallos, 2006). Esas características pueden afectar sus recursos y capacidades.

Las empresas combinan los factores internos y externos (Gilmore \& Carson, 1999) para establecer competencias distintivas (Prahalad \& Hamel, 1990), que les permiten posicionarse mejor que sus competidores. Esas competencias distintivas explican la heterogeneidad en el desempeño de las empresas (Brito \& Brito, 2012), pero el proceso de construcción de dichas competencias tiene elementos similares en todas ellas. Las diferentes características de las empresas pueden condicionar los elementos de competitividad utilizados y la forma de hacer uso de ellos. Este trabajo busca analizar específicamente los factores de construcción de competencias distintivas de pymes madereras en una región de Argentina, y el proceso mediante el cual las competencias distintivas afectan al desempeño de la empresa, con especial referencia al capital intelectual (Chen, Cheng, \& Hwang, 2005).

En particular, se estudia el caso de las pymes madereras situadas en Oberá, una región de la provincia de Misiones en Argentina. El hecho de que el conjunto de pymes estudiadas contengan muchas de las características de clústeres basados en recursos naturales (Giuliani, Pietrobelli, \& Rabellotti, 2005) puede permitir la extensión de algunas de las conclusiones desarrolladas.

En la actual economía del conocimiento, el capital intelectual es considerado fuente de ventaja competitiva en pymes de países desarrollados (Carlucci, Marr, \& Schiuma, 2004) y en países en desarrollo (Jardon \& Martos, 2012). Sin embargo, podría pensarse que, al estar basadas en recursos naturales y ser pymes en una zona, donde principalmente se compite en recursos tangibles (Lindegaard \& Vargas, 2003), el capital intelectual no es importante para estas empresas, Nuestra tesis es que sí es importante, a pesar de que presente particularidades y limi- taciones en relación con otras empresas. Además, su buen uso, les lleva a ser competitivas en el largo plazo. De modo complementario, en este trabajo se estudian otros factores asociados a las competencias distintivas.

Dividiremos el resto del artículo en cuatro apartados. En el primero, se establece el modelo básico de generación de competencias distintivas indicando los constructos que caracterizan sus posibles fuentes e introducimos las proposiciones generales. Después, se establece la metodología. A continuación, se presenta el caso de estudio y se analizan las proposiciones planteadas. Se termina con un apartado de conclusiones.

\section{MARCO CONCEPTUAL}

Muchas pymes latinoamericanas basadas en recursos naturales presentan particularidades. Los empresarios que las dirigen suelen tener poca formación en gestión estratégica (Bruhn, Karlan, \& Schoar, 2010) por lo que suele ser muy limitado el uso de técnicas de gestión profesionalizadas necesitando, en general, un mayor desarrollo de las funciones empresariales. Su gestión se basa, fundamentalmente, en la intuición y la experiencia, con proyectos a mediano plazo (Gatto, 1999). Son individualistas, lo que dificulta la cooperación (Milesi, Moore, Robert, \& Yoguel, 2007), presentando unos altos costes de transacción (Gatto, 1999; Kantis, 1996; Nichter \& Goldmark, 2009). Su pequeño tamaño les limita el acceso a la financiación (Leone, 1991; Wendel \& Harvey, 2006), que junto a la escasa cooperación les lleva a depender de sus propios recursos (Gatto, 1999; Leone, 1991). La preocupación por reducir los costes les condiciona a buscar sus principales fuentes de información en otras empresas (Altenburg \& Meyer-Stamer, 1999) siendo escasas las que recurren a servicios externos de consultoría (Giuliani, Pietrobelli, \& Rabellotti, 2005; Kantis, 1996; Leone, 1991), puesto que no valoran su aporte estratégico. En consecuencia, las decisiones son más intuitivas. Su mercado suele ser restrictivo y, salvo las que se dedican a servicios especializados, se circunscribe a límites geográficos cercanos (Leone, 1991). Al estar basadas en recursos naturales existentes en la zona, suelen presentar una especial habilidad para el manejo de esos recursos. Todo esto sugiere que su estructura de gestión es diferente de las de países desarrollados y posiblemente presente diferencias en cuanto a las competencias distintivas definidas y a sus efectos.

\section{Competencias distintivas en PyMEs}

Las empresas, mediante la estrategia, combinan los recursos y capacidades con los factores clave de la industria establecien- 
do sus competencias distintivas (Grant, 2005), esto es, aquellas cualidades que permiten desarrollar la estrategia de modo superior a la competencia (Prahalad \& Hamel, 1990), por tanto son las que pueden producir el éxito empresarial, tanto a corto como a largo plazo (Ver figura 1).

Figura 1. Modelo de generación de ventajas competitivas en PyMEs basadas en recursos naturales

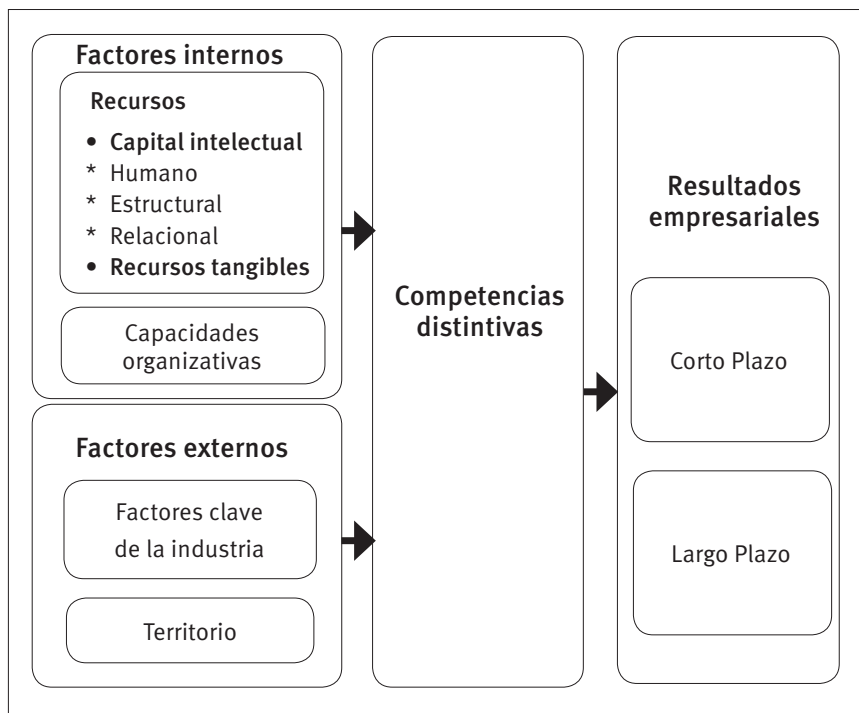

Los factores internos están compuestos de recursos y capacidades. Los recursos pueden ser tangibles o intangibles (Wernerfelt, 1984). Los recursos tangibles son los más fáciles de identificar y evaluar. Incluyen los recursos financieros y los activos físicos identificados y valorados en los estados financieros de la empresa. Los intangibles pueden ser más valiosos pero no aparecen en los estados financieros.

El capital intelectual se refiere a los recursos intangibles que generan valor para la empresa. Presenta tres dimensiones, según el agente donde radica: el capital humano que radica en las personas, el capital estructural que radica en la propia organización y el capital relacional que radica en las relaciones que la organización tiene con el entorno (Bontis, Keow, \& Richardson, 2000).

Las pymes madereras son empresas donde la filosofía del empresario fundador condiciona tanto la cultura como la estrategia de la empresa. Por consiguiente, su capital humano va a ser determinante del éxito empresarial. Estas pymes habitualmente compiten en diferenciación (Porter, 1985). Una gran parte del capital humano de las empresas es específico y, por consiguiente, les permite diferenciarse. Aun cuando les falte formación profesional en relación con la gran empresa, las pymes madereras suelen presentar habilidades en el manejo de esos recursos que les permiten diferenciarse de la competencia, y constituir competencias distintivas, por tanto:

P1: Las pymes madereras desarrollan sus competencias distintivas a partir del capital humano.

Elementos del capital estructural tales como la cultura empresarial (Barney, 1996) o el conocimiento tecnológico (Helfat, 1994) permiten construir competencias distintivas, puesto que facilita diferenciarse de la competencia. Un elemento cultural importante en las pymes madereras está constituido por el saber hacer local, que normalmente está asociado a la madera existente en el territorio y le permite hacer productos más adaptados a los gustos de la zona que otras empresas foráneas. Además, estas pymes suelen estar formadas por grupos pequeños de personas con una cultura similar y una mayor proximidad, lo que mejora la comunicación y, por consiguiente, el clima de trabajo (Wilkinson, 1999), facilitando su coordinación para adaptarse a los cambios del entorno local.

P2: Las pymes madereras desarrollan sus competencias distintivas a partir del capital estructural.

El capital relacional es un elemento clave para competir, puesto que la cooperación facilita encontrar los elementos complementarios para hacer frente a las dificultades del entorno (Humble, Jackson, \& Thomson, 1994), a las limitaciones de financiación (Klofsten \& Scheele, 2003) o a los retos de la innovación (Kantis, 1996). La relación con los proveedores y clientes facilitan la constitución de clústeres que hacen uso de las economías de escala y alcance como factores de competitividad (Felzensztein, Gimmon, \& Carter, 2010). Ese efecto es limitado en las pymes argentinas puesto que evitan los consorcios y no suelen utilizar servicios externos (Kantis, 1996). Sin embargo, su pequeño tamaño les permite una mayor flexibilidad para adaptarse a las necesidades específicas del cliente en cada momento. Además suelen tener conexión directa con clientes y proveedores locales, conociendo sus necesidades por su cercanía. Finalmente, el hecho de compartir una misma cultura con los clientes facilita su relación comercial. Una parte de sus ventas se basa en sus relaciones con los vecinos y en las relaciones de estos con otros conocidos (boca a boca). Todo esto disminuye los costes de transacción, por lo que se espera que su capital relacional sea elemento constitutivo de competencias distintivas.

$P_{3}$ : Las pymes madereras argentinas desarrollan sus competencias distintivas a partir del capital relacional.

Además del capital intelectual, los recursos tangibles de la empresa también son utilizados para competir. En general los 
activos tangibles han sido incluidos entre los factores de competitividad, puesto que se necesitan para mantener su posición competitiva (Mata, Fuerst, \& Barney, 1995; Kaleka, 2002), aunque han sido considerados como coadyuvantes, por el hecho de no ser diferenciables (Balakrishnan \& Fox, 1993). Las pymes, en general, tienen limitaciones de acceso a recursos tecnológicos (Zevallos, 2006) y financieros (Leone, 1991; Wendel \& Harvey, 2006) respecto a las grandes empresas. Las pymes madereras, al estar basadas en recursos naturales se encuentran en la cadena de valor de ese recurso. Esto les permite tener un mejor conocimiento de él, con lo que aprovechan mejor sus propiedades. Además, la cercanía física al recurso reduce los costes logísticos, facilitando constituir competencias distintivas.

P4: Las pymes madereras argentinas utilizan sus recursos tangibles (financieros, tecnológicos y naturales) para constituir sus competencias distintivas.

A diferencia de los recursos, entre los que se incluye el capital intelectual, que son estáticos, las capacidades organizativas son dinámicas y evolucionan en el tiempo (Helfat \& PeteraF, 2003). Helfat y Lieberman (2002) definen capacidad organizativa como la capacidad de una empresa de desplegar recursos para obtener un resultado final deseado. Las capacidades organizativas son muy variadas. Requieren la experiencia de varios individuos para ser integradas con bienes de capital, tecnología y otros recursos, mediante la coordinación de equipos de personas. Las capacidades organizativas se manifiestan en procesos que pueden clasificarse de acuerdo a la cadena de valor puesto que están orientadas a generar valor para el cliente final (Grant, 2005). Las capacidades organizativas presentan particularidades para las pymes. Por ejemplo, la capacidad de gestión es diferente, ya que la práctica directiva es más intuitiva, puesto que los gerentes de pymes suelen tener menos mentalidad estratégica, más orientación al corto plazo, predominio de la cultura orientada a las funciones, dificultad para delegar, insuficiente profesionalización de la gestión y escaso hábito de aprendizaje continuo (Camisón-Zornoza, 1997). Todo esto lleva a constituir capacidades organizativas más sencillas. Esta simplicidad facilita su adaptación a los cambios del entorno, reduciendo los costes asociados. Los procesos organizativos de estas pymes están basados en el saber hacer tradicional, por lo que se ajustan mejor a los mercados tradicionales de la zona generando competencias distintivas que les diferencia de otros emprendedores. Por consiguiente,

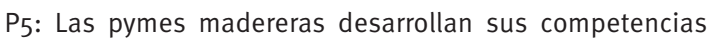
distintivas a partir de las capacidades organizativas.
Entre los factores externos de competitividad, es tradicional incluir en la literatura los factores clave de éxito de la industria (Grant, 2005). Grant (2005) los considera de modo genérico; Zahra y Das (1993) analizan el producto o las estrategias de producción y Carson y Gilmore (2000) estudian el mercado. Las pymes tienen menos capacidad de generar productos y de competir en costes que las grandes empresas pero su cercanía al cliente les permite diferenciarse de un modo más específico y adaptarse mejor a las necesidades del mercado (Pelham, 1997) generando más valor para el cliente. Estas características también se presentan en las pymes madereras. Consecuentemente sugerimos:

P6: Las pymes madereras hacen uso de los factores clave de la industria para construir sus competencias distintivas.

Generalmente las pymes están muy asociadas al territorio. Dada su pequeña dimensión tienen una mayor dependencia del entorno local. El territorio posibilita el éxito de la empresa (Vázquez-Barquero, 1999), ya que la cercanía física facilita un mayor intercambio de relaciones con proveedores y clientes (Camisón-Zornoza, 1997); abarata los costes logísticos, muy importantes en Latinoamérica (Brennan \& Garvey, 2009), y facilitan la generación de economías de aglomeración y de marketing (Felzensztein, Gimmon, \& Carter, 2010). En el caso de las pymes madereras, el territorio es un factor de diferenciación mediante denominaciones de origen o estrategias similares.

P7: Las pymes madereras hacen uso del territorio donde se asienta la empresa para desarrollar sus competencias distintivas.

\section{Competencias distintivas y resultados empresariales}

Las pymes argentinas presentan habitualmente diferencias entre los resultados a largo plazo, que se manifiestan en los resultados organizativos, y a corto plazo, manifestados en los resultados económico-financieros. Varias son las posibles causas de esta discrepancia. Primeramente, la situación de estas empresas que se apoyan más en los recursos materiales que en las capacidades (Lindegaard \& Vargas, 2003); también, la estructura normativa y política del país que, al incrementar el riesgo, limita la competitividad en el largo plazo y dificulta la integración de ambos enfoques. Además, las empresas madereras argentinas tienen un alto porcentaje de productores que utilizan únicamente su negocio como fuente de subsistencia, lo que les lleva a buscar beneficios a corto plazo (Gatto, 1999), sin elaborar estrategias de largo alcance. Finalmente, estas empresas presentan un menor nivel de formación, que les dificulta la planifica- 
ción a largo plazo. Todo ello posibilita la existencia de un mayor oportunismo empresarial, por lo tanto:

P8: Existen diferencias entre los resultados organizativos (con enfoque de largo alcance) y los resultados económicos (de corto plazo) en las pymes madereras argentinas.

Finalmente, nos interesa asegurar que las competencias distintivas generadas producen realmente mejor desempeño. Las competencias distintivas se elaboran para competir en el largo plazo (Grant, 2005), por consiguiente, se espera que los resultados a largo plazo mejoren cuando las empresa cuentan con competencias distintivas. Complementariamente, se analiza si también se produce una mejora en el corto plazo dada la divergencia entre ambos efectos. En consecuencia sugerimos

P9: Las competencias distintivas en pymes madereras argentinas producen mejores resultados empresariales, especialmente en el largo plazo.

\section{METODOLOGÍA Y FUENTES DE INFORMACIÓN}

En primer lugar se analiza la población y muestra del caso seleccionado indicando la metodología del tratamiento de esa información.

\section{Población y muestra}

La información necesaria se obtuvo mediante la realización de encuestas a los propietarios y gerentes de empresas transformadoras de madera ubicadas en el Departamento Oberá, durante finales de 2005 y principios de 2006. El diseño de la muestra se hizo aleatoriamente de modo proporcional a los municipios que conforman el departamento. Se seleccionaron 141 empresas con un error de muestreo del 3\% y un nivel de confianza del $95 \%$, suponiendo una varianza máxima donde $\mathrm{p}$ $=q=0,5$. La tasa de respuesta fue del $83 \%$, por lo que el tamaño final de la muestra fue de 117 empresas, incrementando el error al 5,09\%. Se comprobó que la estructura final de la muestra se ajustaba a la población de estudio mediante un test de homogeneidad de poblaciones (Newbold, Carlson, \& Thorne, 2002).

\section{Medidas}

La forma del cuestionario siguió el esquema de los trabajos de Jardón et al. (2007) basado en escalas contrastadas en la lite- ratura (Deshpande \& Golhar, 1994). Se hicieron dos bloques de preguntas. Para medir los factores de competitividad, se les pidió a los empresarios que valoraran la importancia de cada uno de los ítems (ver Tabla 1) como ventaja competitiva de su empresa con relación a sus competidores, en una escala que iba desde 1 (no es importante) hasta 5 (es muy importante).

Para evaluar el éxito se puede hacer uso de datos cuantitativos o cualitativos. Los datos cuantitativos parecen más objetivos, puesto que están recogidos de forma numérica y son igualmente considerados por todos los observadores. Sin embargo dichos datos están basados en un sistema de información contable determinado por consideraciones legales y fiscales que pueden tergiversar la realidad explicita de la empresa. Además, a pesar de la objetividad de los indicadores financieros, sólo reflejan el rendimiento pasado y no necesariamente apuntan a la sostenibilidad de éxito en el futuro. Finalmente, los estudios basados en datos financieros presentan serias limitaciones en cuanto a disponibilidad de datos (Anand, Brenes, Karnani, \& Rodriguez, 2006). Por eso en diferentes trabajos de investigación se ha optado por datos subjetivos (Lahiri, Kedia, \& Mukherjee, 2012). Siguiendo dicho esquema, se preguntó por el crecimiento de los diferentes ítems respecto a sus competidores (ver Tabla 2 ) en los últimos dos años en una escala de 1 (ha disminuido mucho) a 5 (ha aumentado mucho).

\section{Técnicas estadísticas}

El proceso utilizado tiene varias fases: a) agrupar los ítems que caracterizan cada posible factor de competitividad; b) explorar como se combinan para generar competencias distintivas; c) comprobar si estas competencias promueven el éxito de la empresa.

Para construir los constructos que caracterizan los factores de competitividad y las competencias distintivas utilizaremos el análisis de componentes principales (Hair, Black, Babin, Anderson, \& Tatham, 2006). El número de componentes utilizadas para medir cada constructo se decide mediante el grafico de sedimentación, asegurando que la varianza explicada sea alrededor del 50\% (Costello \& Osborne, 2005). Cuando sea necesario rotar las componentes, haremos una rotación oblimin directa con o, puesto que permite que haya interrelación entre las componentes. El grado de validez viene dado por el test de Bartlett que contrasta si existe algún tipo de información común entre los ítems originales. Para evaluar la inclusión de un ítem en el modelo se hace uso de la comunalidad que indica la parte explicada de ese ítem por el modelo. Si es menor de 0,4 será eliminado (F-Jardon \& Martos, 2011). 


\section{TABLA 1. Fuentes de ventajas competitivas}

\begin{tabular}{|c|c|c|}
\hline Componente & Ítems considerados & Referencias \\
\hline \multirow{6}{*}{ Capital Humano } & La actitud de los directivos y de los trabajadores & (Hornsby \& Kuratko, 2003) \\
\hline & La capacidad de evaluación de riesgos de las inversiones & (Balakrishnan \& Fox, 1993) \\
\hline & $\begin{array}{l}\text { La capacidad de innovación de procesos, productos o } \\
\text { mercados }\end{array}$ & (Klofsten \& Scheele, 2003) \\
\hline & La capacidad de transmitir experiencias & (Jardon et al., 2007) \\
\hline & La formación de los directivos y trabajadores & (Pfeffer, 2005) \\
\hline & Los recursos humanos & (Hatch \& Dyer, 2004) \\
\hline \multirow{5}{*}{$\begin{array}{l}\text { Capital } \\
\text { estructural }\end{array}$} & El conocimiento del mercado & (Carson \& Gilmore, 2000) \\
\hline & El trabajo en equipo & (Coff, 2008) \\
\hline & La comunicación interna & (Barney, 1996) \\
\hline & La cultura de la empresa & (Barney, 1996; Ritchie \& Brindley, 2005) \\
\hline & Las tecnologías de procesos y productos utilizadas & (Helfat, 1994; Kim \& Kogut, 1996) \\
\hline \multirow{7}{*}{$\begin{array}{l}\text { Capital } \\
\text { relacional }\end{array}$} & $\begin{array}{l}\text { La actitud de cooperación y alianzas por parte de la } \\
\text { empresa }\end{array}$ & (Cal bouzada, Verdugo Matés, \& Fernández-Jardón, 2007) \\
\hline & La red de distribución & (Spillan \& Parnell, 2006) \\
\hline & La imagen de la empresa & (Gattermann, Hoffmann, \& Hooley, 2007) \\
\hline & El tipo decanales de distribución & (Anand et al., 2006) \\
\hline & La relación directa con los clientes finales & (Pelham, 1997; Spillan \& Parnell, 2006) \\
\hline & El tipo de clientes & (Pelham, 1997; Spillan \& Parnell, 2006) \\
\hline & El tipo de proveedores & (Wagner, 2006) \\
\hline \multirow{3}{*}{$\begin{array}{l}\text { Recursos } \\
\text { tangibles }\end{array}$} & Los recursos financieros/estructura económico financiera & (Balakrishnan \& Fox, 1993) \\
\hline & Los recursos tecnológicos de instalaciones y equipamientos & (Kim \& Kogut, 1996) \\
\hline & Los recursos naturales & (Porter, 1985; Vázquez-Barquero, 1999) \\
\hline \multirow{9}{*}{$\begin{array}{l}\text { Capacidades } \\
\text { organizativas }\end{array}$} & El sistema de dirección & (Grant, 2005) \\
\hline & El sistema de fidelización de clientes & (Pelham, 1997; Spillan \& Parnell, 2006) \\
\hline & El proceso de investigación/desarrollo/innovación & (Teach, 1990) \\
\hline & El proceso de diseño & (Helfat, 1994) \\
\hline & El sistema de información & (Mata, Fuerst, \& Barney, 1995) \\
\hline & El proceso de aprovisionamiento & (Porter, 1985) \\
\hline & El sistema de producción & (Porter, 1985; Zahra \& Das, 1993) \\
\hline & El proceso de comercialización y venta & (Porter, 1985) \\
\hline & El servicio posventa & (Porter, 1985) \\
\hline \multirow{3}{*}{ Zona } & Las áreas geográficas en las que compra & (Porter, 1985; Vázquez-Barquero, 1999) \\
\hline & Las áreas geográficas en las que produce & (Porter, 1985; Vázquez-Barquero, 1999) \\
\hline & Las áreas geográficas en las que vende & (Porter, 1985; Vázquez-Barquero, 1999) \\
\hline \multirow{7}{*}{$\begin{array}{l}\text { Factores } \\
\text { estratégicos }\end{array}$} & La promoción de los productos/servicios & (Kotler, 1999) \\
\hline & La amplitud de la cartera de productos/servicios & (Kotler, 1999) \\
\hline & La atención al cliente & (Carson \& Gilmore, 2000) \\
\hline & El precio que tienen los productos/servicios & (Spillan \& Ziemnowicz, 2003) \\
\hline & La exclusividad de los productos/servicios & (Kotler, 1999; Zahra \& Das, 1993) \\
\hline & La calidad de los productos/servicios & (Anand et al., 2006) \\
\hline & La disponibilidad de los productos servicios & (Kotler, 1999) \\
\hline
\end{tabular}


TABLA 2. Éxito empresarial

\begin{tabular}{|c|c|}
\hline Items & References \\
\hline Facturación & (Bontis, Keow, \& Richardson, 2000) \\
\hline Cash flow & (Chakravarthy, 1986) \\
\hline Beneficio & (Darroch, 2005) \\
\hline Rentabilidad & $\begin{array}{l}\text { (Chen, Cheng, \& Hwang, 2005; Darroch, } \\
\text { 2005) }\end{array}$ \\
\hline Solvencia & (Katchova, 2010) \\
\hline $\begin{array}{l}\text { Transferencia } \\
\text { tecnológica }\end{array}$ & (Chen, Cheng, \& Hwang, 2005) \\
\hline $\begin{array}{l}\text { Profesionalidad de los } \\
\text { trabajadores }\end{array}$ & (Rangone, 1999) \\
\hline Productividad & (Valmohammadi \& Servati, 2011) \\
\hline Coste de producción & (Valmohammadi \& Servati, 2011) \\
\hline Modernización & (Bontis, Keow, \& Richardson, 2000) \\
\hline $\begin{array}{l}\text { Capacidad de } \\
\text { innovación }\end{array}$ & (Bontis, Keow, \& Richardson, 2000) \\
\hline Valor de mercado & (Darroch, 2005) \\
\hline $\begin{array}{l}\text { Ventajas competitivas } \\
\text { de la empresa }\end{array}$ & (Darroch, 2005) \\
\hline
\end{tabular}

Para establecer la confiabilidad del instrumento de medida y recolección de datos, se calculó el coeficiente Alfa de Cronbach, que determina la consistencia interna del cuestionario diciéndonos si los ítems utilizados para medir un único constructo son coherentes entre sí. Este coeficiente se basa en el análisis de las correlaciones promedios entre los ítems referidos a un mismo aspecto, a partir de una sola administración del cuestionario (Cronbach, 1951). Si el valor es mayor de o,6 se considera adecuada (Nunnally \& Bernstein, 1994).

Posteriormente, para comprobar el modelo de modo conjunto y determinar la validez de las proposiciones para el caso estudiado haremos uso de la técnica de mínimos cuadrados parciales (PLS) que no necesita la suposición de normalidad de las variables, difícilmente verificable en este caso. Las soluciones basadas en PLS intentan minimizar la varianza de to- das las variables dependientes en función de las variables causales. Esta técnica utiliza la varianza media extraída (AVE) y el alfa de Cronbach (CA) -previamente definida- como criterios para validar el modelo. AVE mide la varianza capturada por un constructo latente. Esta cantidad se suele considerar buena si es superior a 0,5. La ley de distribución de los estimadores es desconocida. Por lo tanto, se utilizó una técnica bootstrap para contrastar la significación de los parámetros (Efron, 1979).

El software utilizado para el proceso de análisis exploratorio fue el paquete SPSS (versión 15) y para la determinación del modelo conjunto fue Smart-PLS (Ringle, Wende, \& Will, 2005).

\section{COMPETENCIAS DISTINTIVAS EN LAS PYMES MADERERAS DEL DEPARTAMENTO DE OBERÁ}

La exploración del modelo se llevó a cabo en la industria maderera del Departamento Oberá (“la que brilla”, en lengua guaraní), que está ubicado en la Zona Centro Este de la provincia de Misiones (Argentina) y consta de 9 municipios. Tiene una superficie de $1.620 \mathrm{~km}^{2}$, equivalente al 5,2 \% del total de la provincia. Su población recibió el aporte masivo de inmigrantes de diferentes países. La agricultura está fuertemente orientada a la producción de cultivos a gran escala como es el caso del té, la yerba mate o el tabaco que constituyen el $57 \%$ del total cultivado, seguido por la explotación forestal de bosques y montes tanto nativos como implantados (INDEC, 2002). La industria maderera cuenta con 162 empresas que realizan la primera y segunda transformación de la madera (MernryT, 2003), si bien la mayor parte son aserraderos aunque realizan otras actividades bajo pedido. La actividad maderera se ha potenciado a partir de la crisis de 2001 en Argentina, aunque la mayoría son pequeñas empresas (ver tabla 3).

\section{TABLA 3. Características: Numero de empresas}

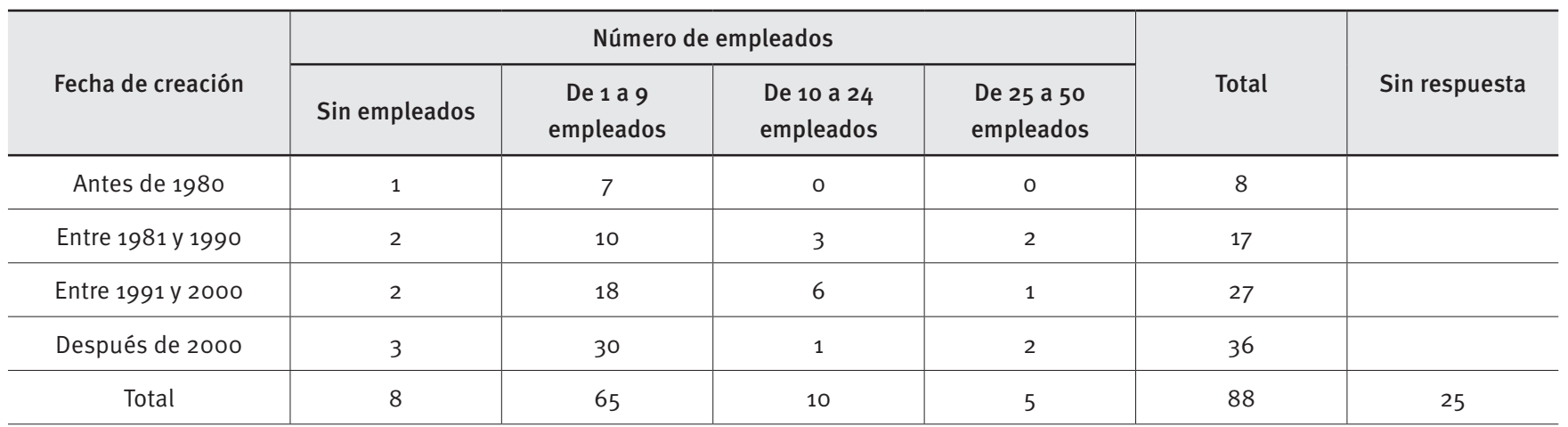


Su insuficiente dinamismo y tamaño ha dificultado el desarrollo de actividades conexas y de proveedores especializados de bienes de capital e insumos intermedios de alguna significación. Los servicios de apoyo y actividades complementarias son casi inexistentes. La tecnología utilizada en maquinarias y equipos es básicamente importada, con pequeñas excepciones (Maslatón, Ladrón \& Gonzalez, 2008). La I+D es casi inexistente en el sector privado, con excepción de algunos desarrollos realizados por grandes empresas en campos como genética, técnicas de manejo y servicios forestales y muy limitado en centros especializados del sector público (UNaM, INTA), por sus escasos recursos.

El análisis previo de componentes principales permitió eliminar ítems poco importantes y asegurar la validez del resto de los ítems para evaluar los diferentes factores de competitividad. El análisis mediante PLS confirma esos hallazgos (ver tabla 4). Dicho análisis sugiere que los empresarios construyen una única competencia distintiva, combinando todos los factores, que denominaremos nivel de competitividad. En ese sentido, estas pymes se diferencian de las pymes de los países desarrollados donde se suelen establecer diferentes competencias distintivas (F-Jardon \& Martos, 2011). Por consiguiente, las políticas de actuación en estas pymes deben ser específicas y, posiblemente, las políticas generales establecidas en otras zonas no sean válidas.

El análisis selecciona dos componentes para representar el desempeño de la empresa. La primera de ellas está relacionada básicamente con medidas asociadas a los resultados económicos de la empresa. La segunda se relaciona con aspectos organizacionales, por lo que la denominaremos resultados organizativos. Por tanto, los resultados empresariales se descomponen en dos factores que representan los resultados económicos y los resultados organizativos de la empresa respectivamente. Uno está más orientado al corto plazo y el otro al largo plazo. De modo individual, presentan baja correlación $(r=0,03)$. Esto parece indicar una divergencia entre los elementos necesarios para desarrollar su competitividad, lo que sugiere la validez de la proposición 8. Por un lado, la empresa debe tener en cuenta los resultados organizativos que permiten la competitividad en el largo plazo y, por otro debe considerar los resultados económicos que conllevan una competitividad en el corto plazo. Por consiguiente, el éxito empresarial aparece medido de modo diferente que en los países desarrollados.

Finalmente, analizamos la estimación del modelo completo mediante técnicas PLS. Los resultados muestran cuales son los elementos más significativos en cada constructo (ver tabla 4) y como son los efectos entre los constructos (ver tabla 5). El capital humano especialmente manifestado en la capacidad de evaluación de riesgos de las inversiones, la capacidad de innovación de procesos, productos o mercados y la formación de los directivos y trabajadores, afecta significativamente al nivel de competitividad, sugiriendo que forma parte de esa competencia distintiva. Esto coincide con otros trabajos en países desarrollados (Hatch \& Dyer, 2004) o en desarrollo (Wood, Tonelli, \& Cooke, 2012).

También podemos afirmar que el capital estructural y, en especial, la comunicación interna, también forma parte de las competencias distintivas. Esto completa los trabajos de Carson y Gilmore (2000), Coff (2008); Barney (1996), Ritchie y Brindley ( 2005) y Kim y Kogut (1996), puesto que sugiere que cada uno de los elementos debe ser combinado con otros para generar ventajas competitivas para la empresa.

De modo similar se asegura que el capital relacional, pero especialmente la relación con los clientes, el tipo de cliente y la red de distribución, afecta significativamente a la competencia distintiva, coincidiendo con los trabajos de Welbourne y Pardo-Del-Val (2008).

Los recursos tangibles y, de modo particular, los recursos financieros, también forman parte de la competencia distintiva elaborada en estas pymes, sugiriendo que para obtener competencias distintivas, es necesaria la financiación combinada con otros elementos, posiblemente por ser escasa en estas pymes (Angelelli, Moudry, \& Llisterri, 2006).

Las capacidades organizativas, con especial referencia a los procesos de aprovisionamiento y de comercialización y venta, junto con los sistemas de fidelización de clientes y de producción, ayudan a constituir la competencia distintiva de estas pymes, coincidiendo con trabajos previos (Teece, Pisano, \& Shuen, 1997).

Los factores clave de éxito de la industria, y, de modo particular, la calidad de los productos/servicios permiten constituir competencias distintivas en las pymes madereras, si bien es necesario combinar esos factores para conseguirlo (Anand et al., 2006; Kotler, 1999).

Finalmente, el territorio, considerado como las áreas geográficas en las que realiza su actividad pero, en especial, las áreas en las que vende, actúa también como elemento constitutivo de competencias distintivas, de acuerdo a las teorías de clústeres empresariales (Felzensztein, Gimmon, \& Carter, 2010).Todos estos resultados nos confirman las proposiciones $\mathrm{P}_{1}$ a $\mathrm{P} 7$ para este caso.

Los cálculos recogidos en la tabla 5 muestran divergencias al analizar la hipótesis de impacto sobre los resultados de la empresa. El efecto sobre los resultados organizativos es significativo, mientras que el efecto sobre los resultados económicos no lo es, por consiguiente, podríamos decir que el nivel de competitividad no produce resultados a corto a plazo pero si está orientada al largo plazo. 
TABLA 4. Cargas factoriales y t-estadísticos bootstrap

\begin{tabular}{|c|c|c|c|c|}
\hline constr & variable & Efecto & Estándar Desviación & T Estadístico \\
\hline \multirow{5}{*}{$\begin{array}{l}\text { Capital humano } \\
\text { AVE }=0,48 \\
C A=0,79\end{array}$} & La actitud de los directivos y de los trabajadores & 0,62 & 0,09 & 6,79 \\
\hline & La capacidad de evaluación de riesgos de las inversiones & 0,74 & 0,06 & 12,68 \\
\hline & La capacidad de transmitir experiencias & 0,61 & 0,13 & 4,77 \\
\hline & La formación de los directivos y trabajadores & 0,77 & 0,06 & 13,08 \\
\hline & Los recursos humanos & 0,68 & 0,08 & 8,72 \\
\hline \multirow{4}{*}{$\begin{array}{l}\text { Capital estructural } \\
A V E=0,35 \\
C A=0,54\end{array}$} & El trabajo en equipo & 0,60 & 0,17 & 3,53 \\
\hline & La comunicación interna & 0,63 & 0,07 & 8,62 \\
\hline & La cultura de la empresa & 0,65 & 0,11 & 5,88 \\
\hline & Las tecnologías de procesos y productos utilizadas & 0,56 & 0,11 & 5,01 \\
\hline \multirow{4}{*}{$\begin{array}{l}\text { Capital relacional } \\
A V E=0,41 \\
C A=0,63\end{array}$} & El tipo de canales de distribución & 0,43 & 0,15 & 2,82 \\
\hline & La red de distribución & 0,75 & 0,07 & 10,49 \\
\hline & La relación directa con los clientes finales & 0,68 & 0,06 & 10,50 \\
\hline & Los acuerdos de cooperación establecidos & 0,57 & 0,09 & 6,29 \\
\hline \multirow{3}{*}{$\begin{array}{l}\text { Recursos tangibles } \\
A V E=0,68 \\
C A=0,76\end{array}$} & Los recursos naturales & 0,57 & 0,12 & 4,76 \\
\hline & Las instalaciones y los recursos tecnológicos & 0,92 & 0,02 & 43,39 \\
\hline & Los recursos financieros & 0,93 & 0,02 & 52,03 \\
\hline \multirow{4}{*}{$\begin{array}{l}\text { Capacidades } \\
\text { organizativas } \\
A V E=0,42 \\
C A=0,80\end{array}$} & El proceso de aprovisionamiento & 0,70 & 0,06 & 11,37 \\
\hline & El proceso de comercialización y venta & 0,70 & 0,07 & 9,76 \\
\hline & El proceso de diseño & 0,64 & 0,11 & 5,71 \\
\hline & El proceso de investigación y desarrollo & 0,54 & 0,13 & 4,26 \\
\hline $\begin{array}{l}\text { Territorio } \\
A V E=0,64 \\
C A=0,73\end{array}$ & Las áreas geográficas en las que vende & 0,87 & 0,04 & 24,56 \\
\hline \multirow{7}{*}{$\begin{array}{l}\text { Factores clave de la } \\
\text { industria } \\
A V E=0,39 \\
C A=0,74\end{array}$} & El precio que tienen los productos/servicios & 0,65 & 0,10 & 6,83 \\
\hline & La amplitud de la cartera de productos/servicios & 0,54 & 0,12 & 4,51 \\
\hline & La atención al cliente & 0,54 & 0,08 & 6,78 \\
\hline & La calidad de los productos/servicios & 0,71 & 0,07 & 10,22 \\
\hline & La disponibilidad de los productos/servicios & 0,66 & 0,12 & 5,50 \\
\hline & La exclusividad de los productos/servicios & 0,60 & 0,10 & 6,16 \\
\hline & La promoción y publicidad de los productos/servicios & 0,63 & 0,08 & 7,74 \\
\hline \multirow{6}{*}{$\begin{array}{l}\text { Resultados } \\
\text { económicos } \\
A V E=0,54 \\
C A=0,84\end{array}$} & Beneficio & 0,76 & 0,13 & 5,74 \\
\hline & Cash flow & 0,79 & 0,08 & 9,40 \\
\hline & Facturación & 0,81 & 0,08 & 10,02 \\
\hline & Productividad & 0,70 & 0,13 & 5,39 \\
\hline & Rentabilidad & 0,75 & 0,12 & 6,36 \\
\hline & Solvencia & 0,67 & 0,10 & 6,43 \\
\hline \multirow{4}{*}{$\begin{array}{l}\text { Resultados } \\
\text { organizativos } \\
A V E=0,52 \\
C A=0,70\end{array}$} & Innovación & 0,76 & 0,11 & 7,25 \\
\hline & Modernización & 0,67 & 0,09 & 7,19 \\
\hline & Transferencia tecnológica & 0,73 & 0,08 & 8,59 \\
\hline & Ventaja competitiva generada & 0,74 & 0,08 & 8,91 \\
\hline
\end{tabular}


TABLA 5. Efectos de las trayectorias y t-estadísticos bootstrap

\begin{tabular}{|c|c|c|c|c|c|}
\hline$C E \rightarrow N C$ & 0,12 & 0,11 & 0,02 & 0,02 & 5,90 \\
\hline$C R \rightarrow N C$ & 0,19 & 0,19 & 0,03 & 0,03 & 6,62 \\
\hline $\mathrm{CO} \gg \mathrm{NC}$ & 0,26 & 0,26 & 0,03 & 0,03 & 8,92 \\
\hline Ter-> NC & 0,09 & 0,09 & 0,02 & 0,02 & 4,40 \\
\hline$F E ~>N C$ & 0,21 & 0,21 & 0,03 & 0,03 & 8,31 \\
\hline
\end{tabular}

Este resultado está asociado al caso de estudio. El hecho de que afecte al largo plazo es fácilmente generalizable, puesto que es común con otros estudios previos. Sin embargo la discrepancia entre resultados y el hecho de que no estén relacionados entre sí, puede ser debido a las condiciones específicas del caso de estudio, aunque hay argumentos lógicos que lo justifican, debido a que estas pymes tienen una mayor orientación hacia el corto plazo, que les puede llevar a un comportamiento miópico (Angelelli, Moudry, \& Llisterri, 2006; Gatto, 1999). La estimación del modelo completo mediante PLS muestra que los resultados organizativos afectan a los resultados económicos. Por tanto, las actuaciones de largo plazo terminan produciendo mejor desempeño en el corto plazo. Esto sugiere la validez de la proposición 9.

\section{CONCLUSIONES E INVESTIGACIONES FUTURAS}

Inicialmente partimos de considerar los recursos tangibles (Wernerfelt, 1984), el capital intelectual en sus tres componentes (Bontis, Keow, \& Richardson, 2000), las capacidades organizativas (Grant, 2005), el territorio (Vázquez-Barquero, 1999) y los factores clave de la industria como factores de competitividad (Grant, 2005). A partir de esos elementos, mediante la estrategia, la empresa genera sus competencias distintivas.

Algunos autores analizan el impacto de cada uno de esos factores sobre los resultados (Bontis, Keow, \& Richardson, 2000; Kaleka, 2002), pero no establecen el proceso mediante el cual se generan las competencias distintivas. Otros autores parten de las competencias distintivas de la empresa y muestran su impacto sobre los resultados, comprobando que son ventajas competitivas de la empresa (Gattermann, Hoffmann, \& Hooley, 2007), pero no establecen como se consiguen esas competencias distintivas a partir de los factores. En este trabajo se propone un modelo que enlaza ambos aspectos, determinando la trayectoria de impactos de los factores de competitividad sobre los resultados de la empresa, aplicado a pymes madereras argentinas, que puede extenderse a países en vías de desarrollo en general.

Las pymes madereras argentinas presentan factores de competitividad específicos. Por ejemplo, el capital intelectual aparece como un factor importante, básicamente en dos aspectos: la formación (Pfeffer, 2005) que se manifiesta en el saber hacer con capacidad de innovación (Klofsten \& Scheele, 2003) basado en las tradiciones locales que le permite diferenciarse de competidores internacionales y las actuaciones con los clientes (Felzensztein, Gimmon, \& Carter, 2010) buscando darles productos de calidad (Anand et al., 2006). Este aspecto especifico del capital relacional, se apoya fundamentalmente en la comunicación interna, en la dinámica de los procesos de la empresa (Teece, Pisano, \& Shuen, 1997) y en el territorio donde venden. Otro aspecto relevante es la necesidad de financiación (Wendel \& Harvey, 2006), para lo cual, las pymes buscan los recursos tangibles e intangibles necesarios para paliarlo. Posiblemente estos aspectos sean generalizables a otras empresas argentinas basadas en recursos naturales.

Las pymes argentinas están orientadas al mediano plazo, lo que genera una discrepancia de objetivos. El caso estudiado sugiere que las empresas deben integrar sus objetivos a corto y a largo plazo, lo cual exige un refuerzo del capital intelectual especialmente en la cooperación, la profesionalización y la for- 
mación. La cooperación para desarrollar ventajas competitivas sistémicas de carácter colectivo (Felzensztein, Gimmon, \& Carter, 2010); la profesionalización para afrontar los retos estratégicos de estas pymes y la formación para adaptarse a las necesidades que demanda la sociedad. Las políticas de los estados que limiten la incertidumbre económica reforzaran ese esfuerzo (Kantis, Ishida, \& Komori, 2002).

\section{Limitaciones y futuras líneas de investigación}

Muchas características del caso son comunes a otras pymes latinoamericanas por lo que posiblemente los resultados sean generalizables, aunque los aspectos culturales específicos de la región y actividad estudiadas pueden limitar esa generalización. En particular, Giuliani et al. (2005) sugiere cuatro modelos de clústeres de empresa. Uno de ellos es el clúster basado en recursos naturales en el que se encuentra el caso de estudio. Los resultados son fácilmente generalizables a otras empresas incluidas en ese modelo de clúster y posiblemente a empresas incluidas en el modelo de producción tradicional. Es más cuestionable su generalización a otros casos.

El territorio ha sido analizado como soporte para los proveedores, la empresa o los clientes y no como fuente de cultura o de mercado laboral, o de legislación, etc..., factores todos ellos de atracción. Una línea futura de investigación iría orientada a tener en cuenta esos aspectos complementarios. Además, los datos utilizados se refieren a un determinado periodo de tiempo, por lo que la utilización de datos de panel permitiría generalizar las conclusiones en el tiempo.

\section{REFERENCIAS}

Altenburg, T. \& Meyer-Stamer, J. (1999). How to promote clusters: policy experiences from Latin America. World Development, 27(9), 1693-1713.

Anand, J.; Brenes, E; Karnani, A, \& Rodriquez, A. (2006). Strategic responses to economic liberalization in emerging economies: lessons from experience. Journal of Business Research, 59(3), 365-371.

Angelelli, P., Moudry, R., \& Llisterri, J. J. (2006). Institutional capacities for small business policy development in Latin America and the Caribbean. IDB WP № MSM-136. Washington.

Balakrishnan, S. \& Fox, I. (1993). Asset specificity, firm heterogeneity and capital structure. Strategic Management Journal, 14(1), 3-16.

Barney, J. B. (1996). The resource-based theory of the firm. Organization Science, 7(5), 469.

Bontis, N., Keow, W. C. C., \& Richardson, S. (2000). Intellectual capital and business performance in Malaysian industries. Journal of Intellectual Capital, 1(1), 85-100.

Brennan, L. \& Garvey, D. (2009). The role of knowledge in internationalization. Research in International Business and Finance, 23(2), 120-133.
Brito, R. P. De, \& Brito, L. A. L. (2012). Vantagem competitiva, criação de valor e seus efeitos sobre o desempenho. RAE-Revista de Administração de Empresas, 52(1), 70-84.

Bruhn, B. M., Karlan, D., \& Schoar, A. (2010). What capital is missing in developing countries? American Economic Review: Papers \& Proceedings, $100(2), 629-633$.

Cal Bouzada, M. I., Verdugo Matés, M. V., \& Fernández-Jardón, C. M. (2007). Internacionalización-cooperación nas pemes de Vigo e da súa área metropolitana. Revista galega de economía, 16(1), p. 173-186, 2007.

Camisón-Zornoza, C. (1997). La competitividad de la PYME industrial española: estrategia y competencias distintivas. Madrid: Civitas.

Carlucci, D., Marr, B., \& Schiuma, G. (2004). The knowledge value chain: how intellectual capital impacts on business performance. Int. J. Tech nology Management, 27(6-7), 575-590.

Carson, D. \& Gilmore, A. (2000). SME marketing management competencies. International Business Review, 9(3), 363-382.

Chakravarthy, B. S. (1986). Measuring strategic performance. Strategic Management Journal, 7(5), 437-458.

Chen, M.-C., Cheng, S.-J., \& Hwang, Y. (2005). An empirical investigation of the relationship between intellectual capital and firms' market value and financial performance. Journal of Intellectual Capital, 6(2), 159-176.

Coff, R. W. (2008). Teamwork as competitive advantage. In: West, M. A., Tjosvold, D., \& Smith, K. G. (Org.). International Handbook of Organizational Teamwork and Cooperative Working. Chichester, UK: John Wiley \& Sons Ltd. pp. 483-499.

Cohen Arazi, M. (2013). Las PyMEs en Argentina y la Región. Acceso 9 set. 2013 en http://www.ieral.org/images_db/noticias_archivos/2502-Presentación Marcos Cohen Arazi.pdf.

Costello, A. B. \& Osborne, J. W. (2005). Best practices in exploratory factor analysis: four recommendations for getting the most from your analysis. Practical Assessment, Research \& Evaluation, 10(7), 1-9.

Cronbach, L. J. (1951). Coefficient alpha and the internal structure of tests. Psychometrika, 16(3), 297-334.

Darroch, J. (2005). Knowledge management, innovation and firm performance. Journal of Knowledge Management, 9(3), 101-115.

Deshpande, S. P. \& Golhar, D. Y. (1994). HRM practices in large and small manufacturing firms: A comparative study. Journal of Small Business Management, 32(2), 49-56.

Efron, B. (1979). Bootstrap methods: another look at the jackknife. The Annals of Statistics, 7(1), 1-26.

Felzensztein, C., Gimmon, E., \& Carter, S. (2010). Geographical co-location, social networks and inter-firm marketing co-operation: the case of the salmon industry. Long Range Planning, 43(5-6), 675-690.

F-Jardon, C. \& MartoS, M. S. (2011). Un método para determinar competencias distintivas en pequeñas y medianas empresas. Revista de Administração da UFSM, 4(2), 195-214.

Gattermann, M., Hoffmann, C., \& Hooley, G. (2007). Impacto dos recursos da empresa na performance de inovação. RAE-Revista de Adminis tração de Empresas, 47(4), 46-58.

Gatto, F. (1999). Mercosur: its challenges to small and medium-sized industrial enterprises in terms of competition. Cepal Review, (68), 61-77.

Gilmore, A. \& Carson, D. (1999). Entrepreneurial marketing by networking. New England Journal of Entrepreneurship, 12(2), 31-38. 
Giuliani, E., Pietrobelli, C., \& Rabellotti, R. (2005). Upgrading in global value chains: lessons from Latin American clusters. World Development, 33(4), 549-573.

Grant, R. M. (2005). Contemporary Strategy Analysis. Fifth ed. New York: John Wiley \& Sons.

Hair, JF, Black, WC, Babin, BJ, Anderson, RE, \& Tatham, R L. (2006). Multivariate Data Analysis. 6th. ed. Upper Saddle River: Pearson Prentice Hall.

Hatch, N. W. \& Dyer, J. H. (2004). Human capital and learning as a source of sustainable competitive advantage. Strategic Management Journal, 25(12), 1155-1178.

Helfat, C. E. (1994). Firm-specificity in corporate applied R\&D. Organization Science, 5(2), 173-184.

Helfat, C. E. \& Peteraf, M. A. (2003). The dynamic resource-based view: capability lifecycles. Strategic Management Journal, 24(10), 997-1010.

Helfat, C. E. \& Lieberman, M. (2002). The birth of capabilities: market entry and the importance of pre-history. Industrial and Corporate Change, 11(4), 725-760.

Hornsby, J. S. \& Kuratko, D. F. (2003). Human resource management in U.S. small businesses: a replication and extension. Journal of Developmental Entrepreneurship, 8(1), 73-92.

Humble, J., Jackson, D., \& Thomson, A. (1994). The strategic power of corporate values. Long Range Planning, 27(6), 28-42.

Idb, I.-A. D. B. \& Eri, E. R. I. (2008). From intervention to empowerment: a new approach to assisting SMEs in Latin America. Whasington: [s.n.]. Acceso 23 Ago / 2013 en http://www.iadb.org/es/publicaciones/detalle,7101.html?id=20832.

Indec (Instituto Nacional de Estadística y Censos). Censo Nacional Agropecuario. (2005). Acceso 4 nov. 2005 en http://www.indec.mecon.ar.

Jardon, C. M, Gutawski, R.S.; Martos, M.S.; Aguilar, C. A., \& Barjas, A. (2007). Visión estratégica de la cadena empresarial de la madera de Oberá, Misiones. Posadas: EDUNAM - Editorial Universitaria de la Universidad Nacional de Misiones.

Jardon, C. M. F. \& Martos, M. S. (2010). Determinación de competencias distintivas en pymes: el caso de Vigo y su área de influencia (España) Visión de futuro, 14(2), 73-90.

Jardon, C. M. \& Martos, M. S. (2012). Intellectual capital as competitive advantage in emerging clusters in Latin America. Journal of Intellectual Capital, 13(4), 462-481.

Kaleka, A. (2002). Resources and capabilities driving competitive advantage in export markets: guidelines for industrial exporters. Industrial Marketing Management, 31(3), 273-283.

Kantis, H. (1996). Inercia e innovación en las conductas estratégicas de las PYMES Argentinas. Elementos conceptuales y evidencias empíricas. Buenos Aires: [s.n.]. Acceso 16 jan. 2013 en http://www.cepal.org/cgibin/getProd.asp?xml=/publicaciones/xml/2/4222/P4222.xml\&xsl=/ argentina/tpl/pgf.xsl\&base=/argentina/tpl/top-bottom.xsl).

Kantis, H., Ishida, M. and Komori, M. (2002), Entrepreneurship in Emerging Economies:The Creation and Development of New Firms in Latin America and East Asia, Whasington, pp. 1-131.

Katchova, A. (2010). Structural changes in U.S. Agriculture: financial performance of farms in transition. Berlin: [s.n.].

Kim, D.-J. \& Kogut, B. (1996). Technological platforms and diversification. Organization Science, 7(3), 283-301.
Klofsten, M. \& Scheele, J. (2003). Innovation and small enterprises in the Third World. International Small Business Journal, 21(3), 354-357.

Kotler, P. (1999). Marketing Management: Millennium Edition (1oth Edition). Prentice Hall.

Lahiri, S., Kedia, B. L., \& Mukherjee, D. (2012). The impact of management capability on the resource-performance linkage: examining Indian outsourcing providers. Journal of World Business, 47(1), 145-155.

Leone, N. (1991). A dimensão física das pequenas e médias empresas (P,M,E's): à procura de um critério homogeneizador. RAE-Revista de Administração de Empresas, 31(2), 53-59.

Lindegaard, K. \& Vargas, L. (2003). Are Central American export specialization patterns “sticky”? CEPAL Review, 79, 37-50.

Maslatón, C. \& Ladrón Gonzalez, A. (2008). Cifras para pensar: estructura productiva de la industria maderera. Argentina Forestal .com, 4(58), 14-16.

Mata, F. J., Fuerst, W. L., \& Barney, J. B. (1995). Information technology and sustained competitive advantage: a resource-based analysis. MIS Quaterly, 19(4), 487-505.

Mernryt. (2003). Primer compendio cuatrienal estadístico sobre el sector foresto industria de la provincia de Misiones. Ministerio de Ecología, Recursos Naturales Renovables y Turismo de Misiones, Posadas.

Milesi, D.; Moori, V; Robert, V. and Yoguel, G. (2007). Desarrollo de ventajas competitivas: pymes exportadoras exitosas en Argentina, Chile y Colombia. CEPAL Review, 92, 25-43.

Newbold, P., Carlson, W. L., \& Thorne, B. M. (2002). Statistics of business and economic. 5 th ed. New York: Prentice Hall.

Nichter, S. \& Goldmark, L. (2009). Small firm growth in developing countries. World Development, 37(9), 1453-1464.

Nunnally, J. \& Bernstein, I. (1994). Psychometric theory. 3th. ed. [S.I.]: McGraw-Hill Humanities/Social Sciences/Languages.

Pelham, A. (1997). Mediating influences on the relationship between market orientation and profitability in small industrial firms. Journal of Marketing Theory and Practice, 5(3), 55-76.

Pfeffer, J. (2005). Producing sustainable competitive advantage through the effective management of people. Academy of Management Executive, 19(4), 95-106.

Porter, M. E. (1985). Competitive Advantage. New York: The Free Press.

Prahalad, C. K. \& Hamel, G. (1990). The core competence of the corporation. Harvard Business Review, 68(3), 79-91.

Rangone, A. (1999). A resource-based approach to strategy analysis in small-medium sized enterprises. Small Business Economics, 12(3), 233248.

Ringle, C. M., Wende, S., \& Will, A. (2005). SmartPLS Release: 2.0 (beta). Hamburg: SmartPLS Internet. Acceso 20 Mayo/2012 en http://www. smartpls.de.

Ritchie, B. \& Brindley, C. (2005). Cultural determinants of competitiveness within SMEs. Journal of Small Business and Enterprise Development, 12(1), 104-119.

Spillan, J. E. \& Parnell, J. (2006). Marketing resources and firm performance among SMEs. European Management Journal, 24(2-3), 236-245.

Spillan, J. E. \& Ziemnowicz, C. (2003). Strategic management in small retail businesses: the case of Guatemala. International Small Business Journal, 21(4), 461-478. 
Teach, R. (1990). Entrepreneurship and marketing: the pricing and distribution link to success. In: Hills, G., Laforge, R., \& Welsch, H. (Org.). Research At The Marketing/Entrepreneurship Interface. Chicago: The University Of Illinois at Chicago. pp. 351-356.

Teece, D. J., Pisano, G., \& Shuen, A. (1997). Dynamic capabilities and strategic management. Strategic Management Journal, 18(7), 509-533.

Valmohammadi, C. \& Servati, A. (2011). Performance measurement system implementation using Balanced Scorecard and statistical methods. International Journal of Productivity and Performance Management, 6o(5), 493-511.

Vázquez-Barquero, A. (1999). Inward investment and endogenous development: the convergence of the strategies of large firms and territories? Entrepreneurship \& Regional Development, 11(1), 79-93.

Wagner, S. M. (2006). A firm's responses to deficient suppliers and competitive advantage. Journal of Business Research, 59(6), 686-695.
Welbourne, T. M. \& Pardo-Del-Val, M. (2008). Relational capital: strategic advantage for Small and Medium-Size Enterprises (SMEs) through negotiation and collaboration. Group Decision and Negotiation, 18(5), 483-497.

Wendel, B. C. B. \& Harvey, M. (2006). SME Credit scoring: key initiatives, opportunities, and issues. Word Bank Bulletin, 10, 1-6.

Wernerfelt, B. (1984). A resource-based view of the firm. Strategic Management Journal, 5(2), 171-180.

Wilkinson, A. (1999). Employment relations in SMEs. Employee Relations, 21(3), 206-217.

Wood, T. J., Tonelli, M. J., \& Cooke, B. (2012). Para onde vai a gestão de pessoas? GV-Executivo, 11(2), 20-24.

Zahra, S. A. \& Das, S. R. (1993). Building competitive advantage on manufacturing resources. Long Range Planning, 26(2), 90-100.

Zevallos, E. V. (2006). Obstáculos al desarrollo de las pequeñas y medianas empresas en América latina. Cuadernos de Difusión, 11(20), 75-97. 